\title{
Experimental Signatures of the Quantum Nature of Radiation Reaction in the Field of an Ultraintense Laser
}

K. Poder, ${ }^{1, \dagger}$ M. Tamburini, ${ }^{2}$ G. Sarri, ${ }^{3, *}$ A. Di Piazza, ${ }^{2}$ S. Kuschel, ${ }^{4,5}$ C. D. Baird, ${ }^{6}$ K. Behm, ${ }^{7}$ S. Bohlen, ${ }^{8}$ J. M. Cole, ${ }^{1}$ D. J. Corvan, ${ }^{3}$ M. Duff, ${ }^{9}$ E. Gerstmayr, ${ }^{1}$ C. H. Keitel, ${ }^{2}$ K. Krushelnick, ${ }^{7}$ S. P. D. Mangles, ${ }^{1}$ P. McKenna, ${ }^{9}$ C. D. Murphy, ${ }^{6}$ Z. Najmudin, ${ }^{1}$ C. P. Ridgers, ${ }^{6}$ G. M. Samarin, ${ }^{3}$ D. R. Symes, ${ }^{10}$ A. G. R. Thomas, ${ }^{7,11}$ J. Warwick, ${ }^{3}$ and M. Zepf ${ }^{3-5}$

${ }^{1}$ The John Adams Institute for Accelerator Science, Blackett Laboratory, Imperial College London, London SW7 2AZ, United Kingdom

${ }^{2}$ Max-Planck-Institut für Kernphysik, Saupfercheckweg 1, D-69117 Heidelberg, Germany

${ }^{3}$ School of Mathematics and Physics, Queen's University Belfast, University Road, Belfast BT7 INN, United Kingdom

${ }^{4}$ Helmholtz Institute Jena, Fröbelstieg 3, O7743 Jena, Germany

${ }^{5}$ Institut für Optik und Quantenelektronik,

Friedrich-Schiller-Universität Jena, Max-Wien-Platz 1, 07743 Jena, Germany

${ }^{6}$ Department of Physics, University of York, Heslington, York, YO10 5DD, United Kingdom

${ }^{7}$ Center for Ultrafast Optical Science, University of Michigan, Ann Arbor, Michigan 481099-2099, USA

${ }^{8}$ Deutsches Elektronen Synchrotron DESY, Hamburg 22607, Germany

${ }^{9}$ Department of Physics, SUPA, University of Strathclyde, Glasgow, G4 ONG, United Kingdom

${ }^{10}$ Central Laser Facility, Rutherford Appleton Laboratory,

Didcot, Oxfordshire OX11 OQX, United Kingdom

${ }^{11}$ Lancaster University, Lancaster LA1 4YB, United Kingdom

(Received 6 February 2018; revised manuscript received 7 May 2018; published 5 July 2018; corrected 23 August 2018)

\begin{abstract}
The description of the dynamics of an electron in an external electromagnetic field of arbitrary intensity is one of the most fundamental outstanding problems in electrodynamics. Remarkably, to date, there is no unanimously accepted theoretical solution for ultrahigh intensities and little or no experimental data. The basic challenge is the inclusion of the self-interaction of the electron with the field emitted by the electron itself-the so-called radiation reaction force. We report here on the experimental evidence of strong radiation reaction, in an all-optical experiment, during the propagation of highly relativistic electrons (maximum energy exceeding $2 \mathrm{GeV}$ ) through the field of an ultraintense laser (peak intensity of $4 \times 10^{20} \mathrm{~W} / \mathrm{cm}^{2}$ ). In their own rest frame, the highest-energy electrons experience an electric field as high as one quarter of the critical field of quantum electrodynamics and are seen to lose up to $30 \%$ of their kinetic energy during the propagation through the laser field. The experimental data show signatures of quantum effects in the electron dynamics in the external laser field, potentially showing departures from the constant cross field approximation.
\end{abstract}

DOI: 10.1103/PhysRevX.8.031004

Subject Areas: Particles and Fields, Plasma Physics

\section{INTRODUCTION}

In the realm of classical electrodynamics, the problem of radiation reaction (RR) is satisfactorily described by the Landau-Lifshitz (LL) equation [1], which has been

\footnotetext{
* Corresponding author. g.sarri@qub.ac.uk

${ }^{\dagger}$ Present address: Deusches Elektronen Synchrotron DESY, Hamburg 22607, Germany.

Published by the American Physical Society under the terms of the Creative Commons Attribution 4.0 International license. Further distribution of this work must maintain attribution to the author(s) and the published article's title, journal citation, and DOI.
}

theoretically demonstrated to be the self-consistent classical equation of motion for a charged particle [1,2]. However, when the electron experiences extremely intense fields, the LL equation may no longer be assumed valid [3]. A full quantum description is thus required, and this is currently the subject of active theoretical research (see, for instance, Refs. [3-10]). Purely quantum effects can be triggered in these conditions, including the stochastic nature of photon emission [5,6], a hard cutoff in the maximum energy of the emitted photons [9], and pair production [10]. Besides the intrinsic fundamental interest in investigating this regime in laboratory experiments, RR is often invoked to explain the radiative properties of powerful astrophysical objects, such as pulsars and quasars $[11,12]$. A detailed characterization of RR is also important 
for a correct description of high-field experiments using the next generation of multipetawatt laser facilities, such as the Extreme Light Infrastructure [13,14], Apollon [15], Vulcan 20PW [16], and XCELS [17], where focused intensities exceeding $10^{23} \mathrm{~W} / \mathrm{cm}^{2}$ are expected.

The LL equation is obtained assuming that the electromagnetic field in the rest frame of the electron is much smaller than the classical critical field $F_{0}=$ $4 \pi \epsilon_{0} m_{e}^{2} c^{4} / e^{3} \approx 1.8 \times 10^{20} \mathrm{~V} / \mathrm{m}$ [1] and constant over distances of the order of the classical electron radius $r_{0}=e^{2} / 4 \pi \epsilon_{0} m_{e} c^{2} \approx 2.8 \times 10^{-15} \mathrm{~m}$. These conditions are automatically satisfied in classical electrodynamics since quantum effects are negligible as long as the rest frame fields are much smaller than the critical field of quantum electrodynamics (QED) $F_{\text {cr }}=\alpha F_{0} \approx 1.3 \times 10^{18} \mathrm{~V} / \mathrm{m} \ll$ $F_{0}$ [9] and remain constant over distances of the order of the reduced Compton wavelength $\lambda_{C}=r_{0} / \alpha \approx 3.9 \times$ $10^{-13} \mathrm{~m} \gg r_{0}(\alpha \approx 1 / 137$ is the fine structure constant $)$. An electric field with amplitude of the order of the critical field $F_{\text {cr }}$ is able to impart an energy of the order of $m c^{2}$ to an electron over a length of the order of $\lambda_{C}$. If the amplitude of the laser field in the rest frame of the electron is of the order of $F_{\text {cr }}$, the quantum recoil undergone by the electron when it emits a photon is, thus, not negligible [10]. Also, if the laser wavelength in the rest frame of the electron is of the order of $\lambda_{C}$, then already the absorption of a single laser photon would impart to the electron a recoil comparable with its rest energy. Even for $\mathrm{GeV}$ electrons with Lorentz factor $\gamma_{e} \gtrsim 2000$, the micron-scale wavelength of typical high-power laser systems $\left(\lambda_{L} \approx 0.8-1 \mu \mathrm{m}\right)$ implies that the only relevant condition on classicality is on the laser field amplitude $F_{L}$, which, for a plane wave, can be expressed by stating that the quantum parameter $\chi \approx$ $(1-\cos \theta) \gamma_{e} F_{L} / F_{\text {cr }}$ has to be much smaller than unity. Here, $\theta$ is the angle between the laser propagation direction and the electron momentum in the laboratory frame. Thus, the validity of the LL approach can be expected to break down when quantum effects on the electron's motion become important, i.e., when $\chi$ becomes a sizable fraction of unity. In the intense fields that can be created by modernday lasers, one must also account for the possibility of multiple laser photons being absorbed and resulting in the emission of a single high-energy photon by the electron. For each photon formation length, the number of absorbed photons per electron is of the order of the laser dimensionless amplitude $a_{0}=e F_{L} \lambda_{L} / 2 \pi m_{e} c^{2}$ [10]. Available lasers can now easily reach $a_{0} \gg 1$, thus allowing for experimental investigations of this strong-field quantum regime.

The multi-GeV electrons available at accelerator laboratories worldwide would provide an excellent basis for RR studies in the nonlinear and quantum regime, but are rarely available concurrently with ultraintense lasers. The development of compact laser-driven wakefield accelerators (LWFA) [18] provides a well-suited alternative, since it allows $\mathrm{GeV}$ electron beams to be generated directly at high power laser laboratories capable of achieving field strengths of $a_{0} \gg 1$ [19-21]. The plausibility of such an experimental approach is evidenced by the observation of nonlinearities in Compton scattering in previous experimental campaigns [22-24], motivating the study reported here.

To date, only one laser-based experimental campaign has reached a sizable fraction of the Schwinger field in the rest frame of an electron $(\chi \approx 0.2)[25,26]$. While these experiments gave evidence of nonlinearities in Compton scattering [25] and generation of electron-positron pairs [26], no measurements were performed to directly assess the level of RR in the spectrum of the scattered electron beam. Moreover, despite the high field achieved in the electron rest frame, the relatively low intensity of the scattering laser $\left(a_{0} \approx 0.3-0.4\right)$ implies that single photon absorption was the dominant absorption mechanism in the electron dynamics in the field. In other words, nonlinearities only occurred perturbatively; the relative strength of the emission of the $n$th harmonic scales as $a_{0}^{2 n}$, implying that nonlinear Compton scattering was strongly suppressed. In our experimental configuration, a much higher laser intensity $\left(a_{0} \simeq 10\right)$ allowed a strongly nonlinear regime of RR to be accessed (i.e., multiphoton absorption even within a single photon formation length).

We report here on substantial energy loss (up to 30\%) experienced by a laser-driven multi-GeV electron beam (maximum Lorentz factor $\gamma_{e}>4 \times 10^{3}$ ) [27] during its propagation through the focus of a high-intensity laser (dimensionless amplitude $a_{0} \approx 10$ ). A stable regime of laser-driven electron acceleration, obtained using gas-cell targets, allowed us to directly compare the spectrum of the electrons before and after the interaction with the laser. This provides a detailed test of different models of radiation reaction in an electric field that is a sizable fraction (up to $25 \%$ ) of the Schwinger field, distinguishing these results from others recently published in the literature [28]. Best agreement with the experimental data is found for a semiclassical model that weights the LL equation with the ratio between the quantum and classical synchrotron emission spectrum (coefficient of determination $R^{2}=96 \%$, against $R^{2}=87 \%$ for the $\mathrm{LL}$ ), indicating the emergence of quantum effects in the electron dynamics. A residual mismatch between the semiclassical model and the experimental data at low energies could be explained by a potential departure from the realm of validity of the constant cross field approximation (CCFA), an approximation commonly used in modeling the quantum emission of an electron in an external electromagnetic field.

\section{EXPERIMENTAL SETUP}

The experimental setup is shown schematically in Fig. 1(a). One of the twin laser beams of the Astra Gemini laser system [driver laser in Fig. 1(a)], was focused at the entrance of a helium-filled gas cell in order to accelerate a multi-GeV electron beam, via the laser 
(a)

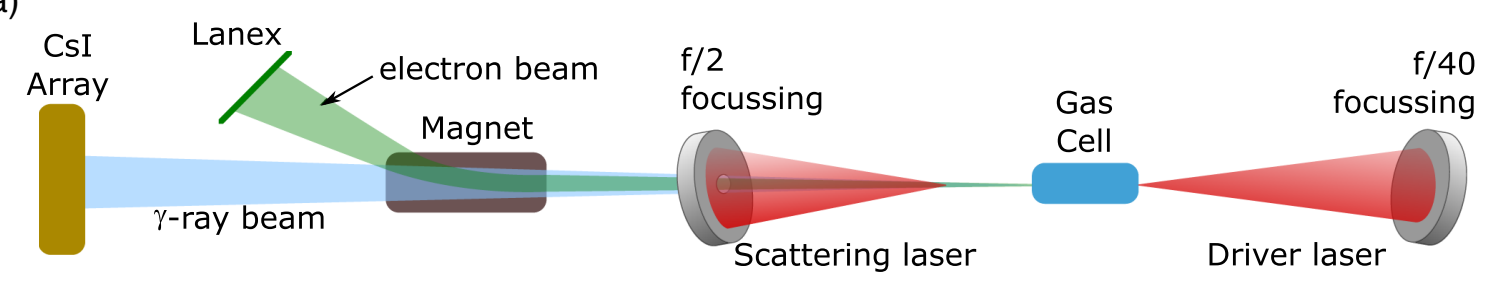

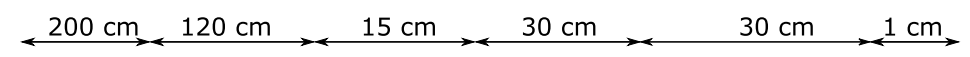

(b)
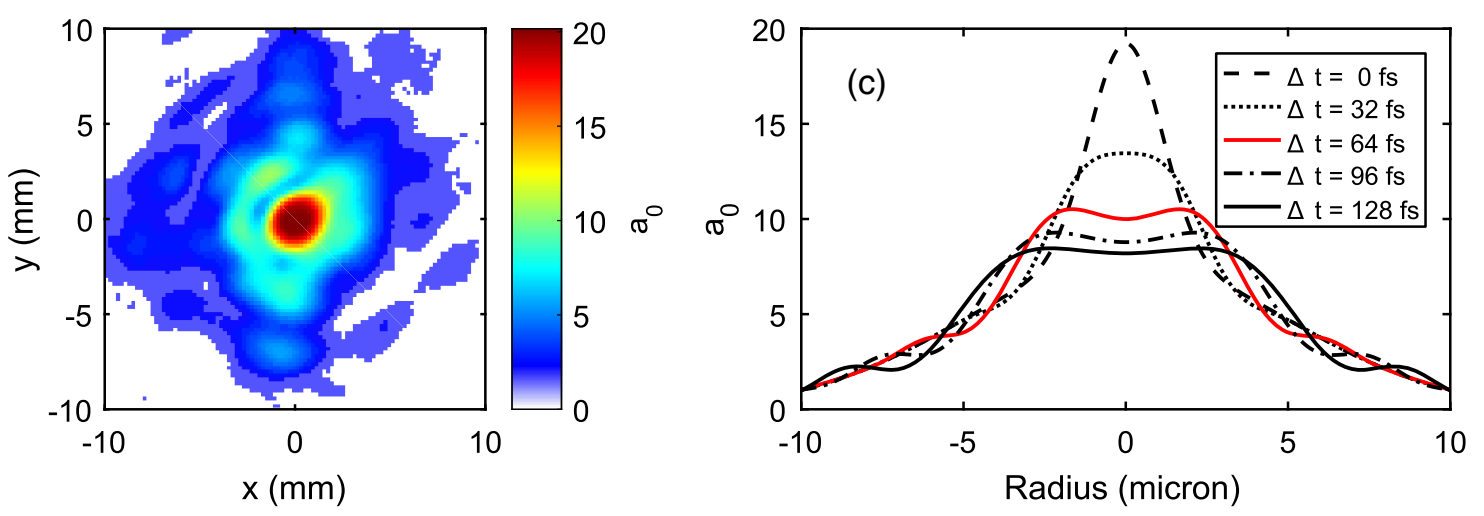

FIG. 1. Experimental setup. (a) Schematic of the experimental setup (not to scale). See details in the text. (b) Typical measured spatial distribution of the intensity in focus of the scattering laser beam. (c) Computed transverse distribution of the normalized laser field amplitude of the scattering laser at the overlap point as a function of time.

wakefield acceleration mechanism [18,27]. The gas cell was operated at a backing pressure of 60 mbar that, once fully ionized, corresponds to an electron density of $2 \times 10^{18} \mathrm{~cm}^{-3}$. The laser with a pulse duration of $(40 \pm 3)$ fs was focused using an $f / 40$ spherical mirror down to a focal spot with a full width at half maximum (FWHM), along the two axes, of $\sigma_{x}=(59 \pm 2) \mu \mathrm{m}$ and $\sigma_{y}=(67 \pm 2) \mu \mathrm{m}$ containing $9 \mathrm{~J}$ (normalized intensity of $a_{0} \approx 1.7$ ).

The laser-driven wakefields in the plasma accelerated the electron beam in the blow-out regime [18], producing stable beams with a broad energy spectrum exceeding $2 \mathrm{GeV}\left(\gamma_{e} \approx 4 \times 10^{3}\right)$ [27]. The electron spectra were recorded by a magnetic spectrometer consisting of a $15-\mathrm{cm}$-long dipole magnet with a peak magnetic field of $1.0 \mathrm{~T}$ and a LANEX scintillator screen placed $2 \mathrm{~m}$ away from the gas cell. The minimum electron energy recorded on the LANEX screen in this configuration was $350 \mathrm{MeV}$, and its energy resolution is of the order of $\delta E / E \approx 5 \%$ for an electron energy of $1.5 \mathrm{GeV}$.

The electron beam source size can be estimated to be $D_{e} \leq 1 \mu \mathrm{m}$, as deduced by rescaling the size of typical betatron sources in similar conditions [29]. The energydependent beam divergence was determined by measuring the beam width perpendicular to the direction of dispersion on the electron spectrometer screen $2 \mathrm{~m}$ downstream from the gas cell. For electron energies exceeding $1 \mathrm{GeV}$, the divergence is measured to be $\theta_{e}=(0.70 \pm 0.05) \mathrm{mrad}$. Even though this gives, in principle, only the divergence along one of the transverse dimensions of the beam, the regime of laser wakefield we are operating in generates accelerating fields with a radially symmetric distribution [18]. This in turn results in cylindrically symmetric electron beams, as confirmed by our analysis [30]. The detailed energy-dependent divergence measured in the experiment was used as an input for the numerical simulations discussed later in the article. Measurements of the pointing fluctuation of the laser-driven electron beam indicate, as an average over 100 consecutive shots, an approximately Gaussian distribution (confidence of 95\% from the Kolmogorov-Smirnov test) centered on the laser propagation axis with a standard deviation of $(3.2 \pm 0.8) \mathrm{mrad}$ [30]. The use of a gas-cell target, instead of a gas jet reported elsewhere [28] for similar experimental conditions, results in better shot-to-shot stability in the electron spectrum [31,32], with the maximum energy of the electrons closely related to the energy of the drive laser, as discussed in the next section. Moreover, it allowed much higher electron energies to be reached and, therefore, a much higher fraction of the Schwinger field in the electron rest frame.

The second laser beam [scattering laser in Fig. 1(a)] was focused, using an $f / 2$ off-axis parabola with a concentric $f / 7$ hole (energy loss of 10\%), $1 \mathrm{~cm}$ downstream of the exit 
of the gas cell exactly counterpropagating with respect to the laser-wakefield accelerated electron beam. On-shot measurements of the laser temporal profile using a frequency resolved optical gating (FROG) device indicate a Gaussian distribution with a duration of $(42 \pm 3)$ fs. The energy contained in the laser after compression was measured, for each shot, by integrating the beam nearfield on a camera that was previously absolutely calibrated against an energy meter, giving a value of $(8.8 \pm 0.7) \mathrm{J}$. The radial distribution of the laser intensity at focus is shown in Fig. 1(b), and it arises from an average of ten consecutive measurements at low power (spatial resolution of the detector of $0.2 \mu \mathrm{m} /$ pixel). Independent measurements of the intensity profile at low power and full power indicate a broadening of the focal spot radius of the order of $10 \%$ in the latter case [33]. This effect is taken into account in the computed transverse laser field distribution shown in Fig. 1(c).

The scattering and driver laser are linearly polarized along perpendicular axes (horizontal and vertical, respectively) in order to further reduce risks of backpropagation of the lasers in the amplification chains. However, numerical simulations show that the particular polarization axes used in the experiment are virtually irrelevant in determining the energy loss experienced by the electrons. Both lasers are generated from the same oscillator and synchronized using a spectral interferometry technique discussed in Ref. [34] and already used in a similar experimental setup [22]. This system had a temporal resolution of approximately $40 \mathrm{fs}$. Because of the inherent lag of the laser-accelerated electron beam in respect to the driver laser, the scattering laser has defocused for approximately 64 fs before interacting with the electrons [18,27]. At this time delay, the scattering laser has a rather flat profile, with a peak $a_{0}$ of the order of 10 and a full width at half maximum of $7 \mu \mathrm{m}$ [see Fig. 1(c)].

The energy contained in the Compton-generated $\gamma$-ray beam was measured using a 5-cm-thick caesium-iodide (CsI) scintillator placed, on-axis, $4 \mathrm{~m}$ downstream of the electron-laser interaction point. The transverse diameter of each scintillation rod is $5 \mathrm{~mm}$, implying an angular resolution of the order of $1.25 \mathrm{mrad}$. The energy deposited on the scintillator, modeled with FLUKA [35] simulations, is almost linear in the range $10-400 \mathrm{MeV}$ and best fitted $\left(R^{2}=95 \%\right)$ by $E_{\mathrm{DEP}}=2.08 \times 10^{-2} E_{\mathrm{INC}}+0.68$ with $E_{\mathrm{DEP}}$ and $E_{\mathrm{INC}}$ the deposited energy and the energy of the incident photon, respectively.

\section{ELECTRON-LASER OVERLAP AND STABILITY}

One of the main measurables to experimentally assess the amount of RR experienced by the electron beam is the change in spectral energy density from a typical reference electron spectrum to the spectrum of the scattered electrons. In our experiment, the laser-driven electron beams [27] were obtained in a stable regime where their spectral shape was a reproducible function of the input laser energy (Fig. 2), unlike results recently reported using a gas-jet target [28].

In Fig. 2(a), we show the correlation between the energy of the laser driving the wakefield and the cutoff energy of the accelerated electron beam. The cutoff energy is defined as the energy at which the beam spectral intensity falls down to $10 \%$ of its peak value. The empty squares depict shots with the scattering laser off with a linear fit represented by the dashed blue line. The vast majority of these shots fall within $1 \sigma(68 \%$ confidence, darker blue band in the figure), with all of them still within a $2 \sigma$ band (95\% confidence, lighter blue band in the figure). The color-coded circles depict instead shots with the scattering laser on. The color of each circle represents the total energy of the photon beam emitted via Compton scattering, as recorded by the CsI scintillator, normalized by the total kinetic energy in the recorded electron beam (kinetic energy exceeding $350 \mathrm{MeV}$, lower limit of the magnetic spectrometer). As discussed above, the energies of both the driver and scattering laser were measured live on each shot, allowing us to clearly identify suitable reference shots (scattering laser off) for each shot with the scattering laser on.

The intrinsic shot-to-shot pointing fluctuations of LWFA beams [30] result in a statistical fluctuation of the spatial overlap of the laser spot with the electron beam. To discern between shots of poor and good overlap, we use the energy contained in the Compton $\gamma$-ray beam generated during the interaction, an established method for this class of experiments (see, for instance, Ref. [25]). The total energy emitted via Compton scattering scales as $E_{\mathrm{ph}} \propto \int a_{0} \gamma_{e}^{2} N_{e}\left(a_{0}\right) d a_{0}$, with $N_{e}\left(a_{0}\right)$ the number of electrons interacting with a field of amplitude $a_{0}$ [36]. While the CsI detector did not allow for the extraction of the spectral distribution of the photon beam, the signal recorded is proportional to the total energy contained in the Compton-scattered photon beam, allowing us to discern between shots with best overlap (and, therefore, both higher energy loss in the electron beam and high photon yield) from those with poorer overlap. This is exemplified in Fig. 3(a), where the total photon yield recorded on the CsI detector is plotted against the percentage of energy loss experienced by the electron beam. The data appear to follow a linear trend, which is also reproduced by numerical simulations assuming different transverse misalignments of the electron beam with respect to the main axis of the scattering laser. These simulations are performed using a semiclassical model of radiation reaction since, as will be discussed in the following, this is the model that best reproduces our experimental data. This correlation allows us to distinguish between shots with 

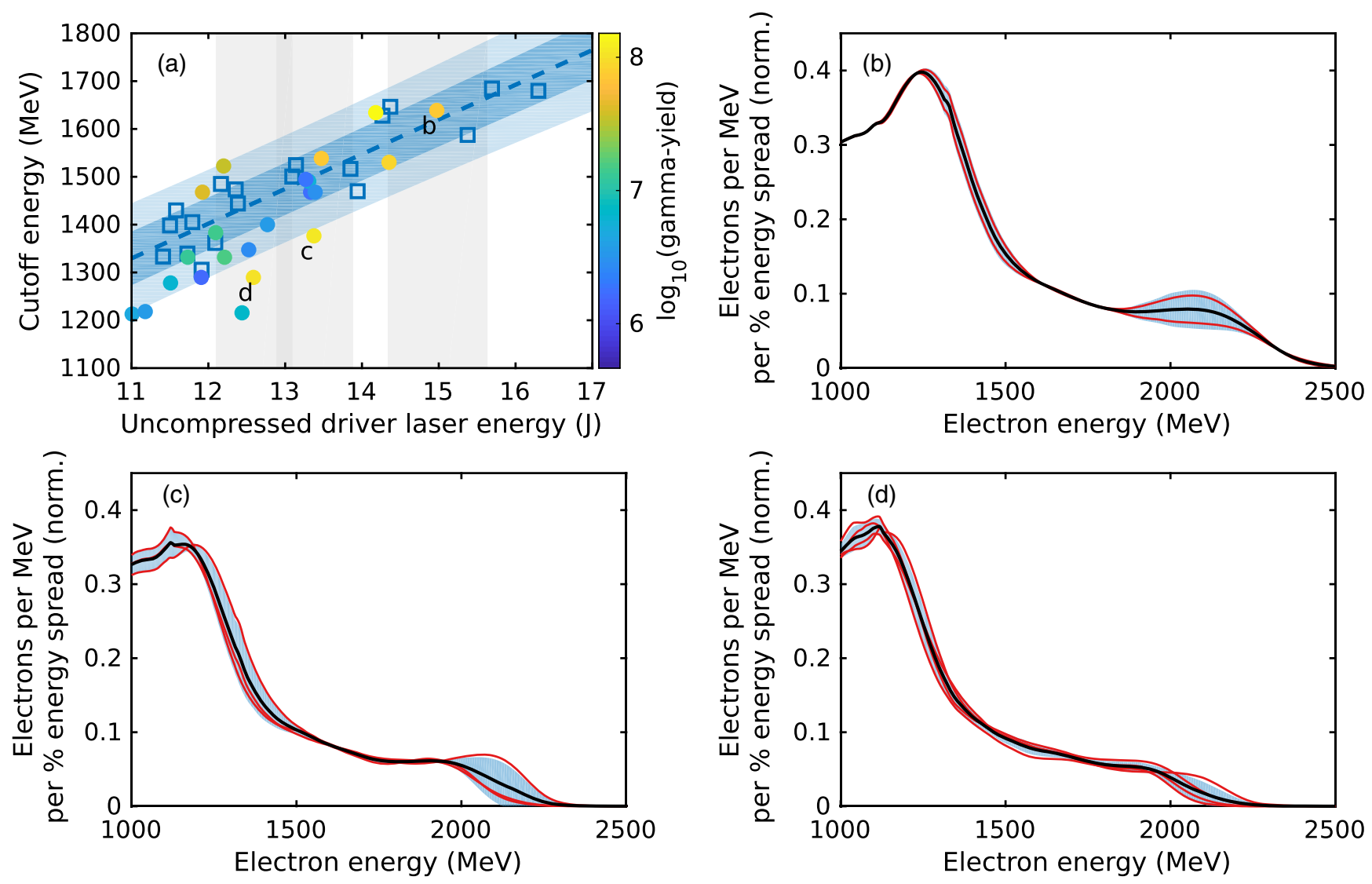

FIG. 2. Reference electron spectra. (a) Cutoff energy of the electron beam for shots with the scattering laser off (reference shots, empty squares) and on (color-coded circles). The dashed blue line represents a linear fit $\left(R^{2}=0.85\right)$ for the reference shots with the lighter and darker blue bands representing regions of $95 \%$ and $68 \%$ confidence, respectively. The circles are colored according to the recorded total energy of the emitted photon beam (colorbar on the right, arbitrary units). The shots analyzed in the manuscript showing strong (d), weak (c), and negligible (b) radiation reaction are also labeled. The grey bands represent regions from where the reference shots for each of the analyzed shots have been selected. (b) Initial electron spectra (scattering laser off) for a laser energy between 14.2 and $15.7 \mathrm{~J}$. (c) Initial electron spectra (scattering laser off) for a laser energy between 12.9 and $13.9 \mathrm{~J}$. (d) Initial electron spectra (scattering laser off) for a laser energy between 12.1 and 13.1 J. In frames (b)-(d), thin red lines represent single shots, thick black lines represent an average, and the associated bands represent one standard deviation.

good overlap [labeled c and d in Fig. 3(a)] from shots with poor overlap [such as the shot labeled b in Fig. 3(a)]. Indeed, shots with relatively low photon yield all fall within the $2 \sigma$ band (lighter blue band) of the linear dependence of the electron beam cutoff energy on the energy of the driver laser. On the other hand, the two shots with the brightest photon signal [labeled with $d$ and $c$ in Fig. 2(a)] both fall outside the $2 \sigma$ band, implying that the probability of them being just the result of a random fluctuation is smaller than $0.2 \%$. This places high confidence that a measurement of a lower electron energy is directly related to the occurrence of strong RR.

In the following, we will then focus on three exemplary laser shots: the shot labeled $\mathbf{d}$ in Fig. 2(a), a good candidate for best overlap; shot $\mathbf{c}$ as a a good candidate for a slight misalignment between the scattering laser and the electron beam; and shot $\mathbf{b}$ as a good candidate for poor overlap and, therefore, negligible RR. For each of these shots, we have selected the spectra of the primary electron beam whose driver laser energy falls within $0.5 \mathrm{~J}$ [grey bands in Fig. 2(a)] of that of the shot under interest, as reference spectra. The associated spectral densities are plotted in Figs. 2(b)-2(d). For each of these frames, the thin red lines represent single-shot spectral densities, the thick black lines represent the average, and the associated bands represent one standard deviation. As one can see, within each energy band of the driver laser energy, the electron spectral densities were remarkably stable, justifying their use as reference electron spectra for each event with the scattering laser on. In the following, our analysis will be based on single-electron spectra normalized by dividing the measured spectrum by the overall number of electrons with energy exceeding $350 \mathrm{MeV}$, in order to eliminate shot-to-shot fluctuations in the total electron number without affecting the spectral shape of the beam. 

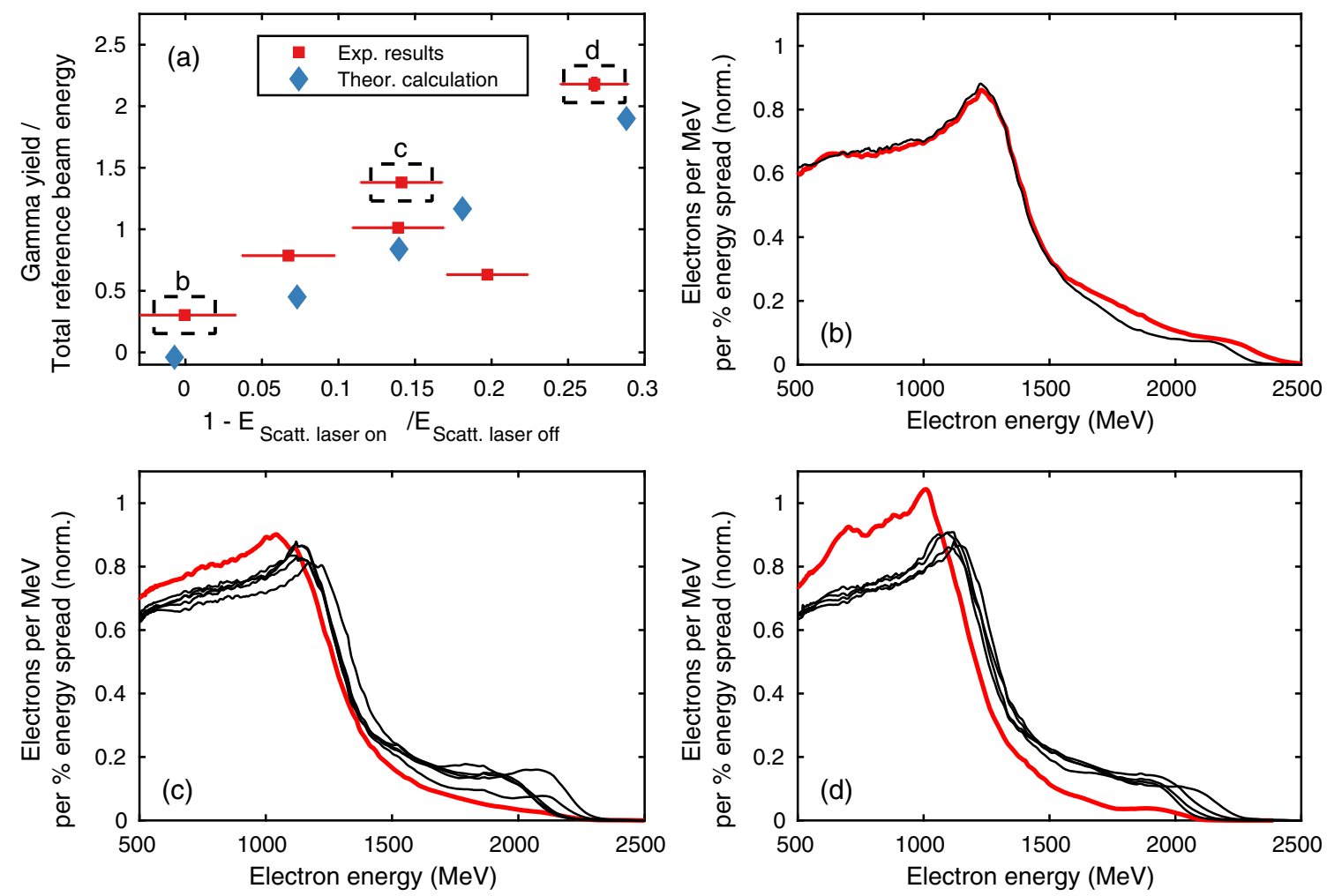

FIG. 3. Radiation reaction data. (a) Measured integrated $\gamma$-beam photon energy (background subtracted and normalized to the total kinetic energy in the unscattered electron beam) versus the amount of radiation friction experienced by the electron beam. Total friction is estimated by dividing the total kinetic energy in the scattered electron beam by the total kinetic energy in the related reference shot. (b)-(d) Measured electron spectrum after interaction with the scattering laser (thick red line) and related spectra with the scattering laser off (black thin line) for the three different scenarios shown in frame (a): poor overlap [frame (b)], moderate overlap [frame (c)], and best overlap [frame (d)].

\section{ELECTRON ENERGY LOSS: EXPERIMENTAL RESULTS}

We will now focus our attention only on shots where the CsI detector indicates best overlap between the high-energy component of the electron beam and the scattering laser [shots $\mathbf{c}$ and $\mathbf{d}$ in Fig. 3(a)]. A comparison between the measured spectral energy density of the initial (scattering laser off) and scattered (scattering laser on) electron beam for conditions of best overlap [shot $\mathbf{d}$ in Fig. 2(a)] is shown in Fig. 3(d). The corresponding single-shot spectral energy densities and the associated uncertainties for the reference electron beams are shown in Fig. 2(d) and exhibit a spectral profile that decreases with energy up to $2 \mathrm{GeV}$, with a clear spectral peak at approximately $1.2 \mathrm{GeV}$. The spectral energy density of the electrons after the interaction with the scattering laser beam [red line in Fig. 3(d)] not only shows a reduction in the cutoff energy but also a significant change in spectral shape, with virtually no electrons with an energy exceeding $1.6 \mathrm{GeV}$. Moreover, the local maximum in the spectrum is now shifted down to an energy of approximately $1 \mathrm{GeV}$, and there is clear accumulation of electrons at lower energies, suggesting a net energy loss for the highest-energy electrons of the order of $30 \%$. On the other hand, a comparison between the scattered and reference electron spectral density for a shot with lower yield [labeled as $\mathbf{c}$ in Fig. 2(a)] clearly evidences a lower amount of energy loss [of the order of 20\%, frame 3(c)], whereas a typical shot with even lower photon yield shows virtually no loss in the electron energy [frame 3(b)].

As a first remark, it is interesting to note that the overall electron energy loss, observed for conditions of best overlap, is slightly lower than a classical estimate based on the LL equation. For our experiment, we can assume a plane wave with a Gaussian temporal field profile given by $\exp \left(-\varphi^{2} / \sigma_{\varphi}^{2}\right)$, where $\varphi=\omega_{L}(t-z / c)$ is the laser phase, $\omega_{L}$ is the laser angular frequency, and $\sigma_{\varphi}=\omega_{L} t_{L} / \sqrt{2 \log 2}$. Here, $t_{L}$ represents the FWHM of the laser intensity. In this case, and assuming $\gamma_{e} \gg a_{0}$, the analytical solution of the LL equation [37] provides

$$
\frac{\Delta \gamma_{e}}{\gamma_{e}} \approx \frac{\sqrt{\pi / \log 2} \tau_{0} t_{L} \omega_{L}^{2} \gamma_{e} a_{0}^{2} / 2}{1+\sqrt{\pi / \log 2} \tau_{0} t_{L} \omega_{L}^{2} \gamma_{e} a_{0}^{2} / 2},
$$

with $\tau_{0}=2 r_{0} / 3 c \approx 6.3 \times 10^{-24} \mathrm{~s}, t_{L}=42 \pm 3$ fs the laser duration, and $\omega_{L}=2.4 \times 10^{15} \mathrm{rad} / \mathrm{s}$ the laser carrier frequency (see also Ref. [38], where there $t_{L}$ corresponds to 
$\sigma_{\varphi} / \omega_{L}$ in our notation). For $\gamma_{e}=4000$ and $a_{0}=10$, the LL equation predicts an energy loss of about $40 \%$, slightly higher than the experimental findings. We observe that under the present experimental conditions (ultrarelativistic electrons with $\gamma_{e} \gg a_{0}$ and initially counterpropagating with respect to the laser field), it is possible to approximate $\gamma_{e} \approx \gamma_{e}\left(1-v_{e, z} / c\right) / 2$, with $v_{e, z} \approx-c$ being the electron velocity along the propagation direction of the laser field, and thus, use directly Eqs. (8) and (9) in Ref. [37] to estimate the relative energy loss. However, in order to provide a more detailed comparison with the different theoretical models of RR, an extensive series of simulations was performed assuming different radiation reaction models and will be discussed in the next section.

\section{ELECTRON ENERGY LOSS: COMPARISON WITH THEORY}

A quantitative comparison between the experimental data and different theoretical models of RR is shown in
Fig. 4. Here, the normalized experimental spectral energy density of the scattered electrons in conditions of best overlap is compared with the corresponding theoretical curves obtained by simulating the effect of the scattering laser on reference spectra using different models and both a multiparticle code and a particle-in-cell (PIC) code. For each frame in the figure, the error bands of the multiparticle code correspond to the uncertainties in the reference electron spectra as well as uncertainties in the intensity of the scattering laser measured for each shot $\left(\Delta a_{0} / a_{0} \simeq 4 \%\right)$.

The multiparticle code assumes a beam of $10^{7}$ electrons generated by sampling first from the experimental electron beam spectrum and then from the energy-dependent divergence, assumed to follow a Gaussian distribution with zero mean and FWHM extracted from the experimental data. The electron three-dimensional momentum was then calculated from the sampled electron energy and from the two sampled divergence angles. In order to account for the free electron propagation from the gas cell, the initial transverse electron spatial distribution was obtained
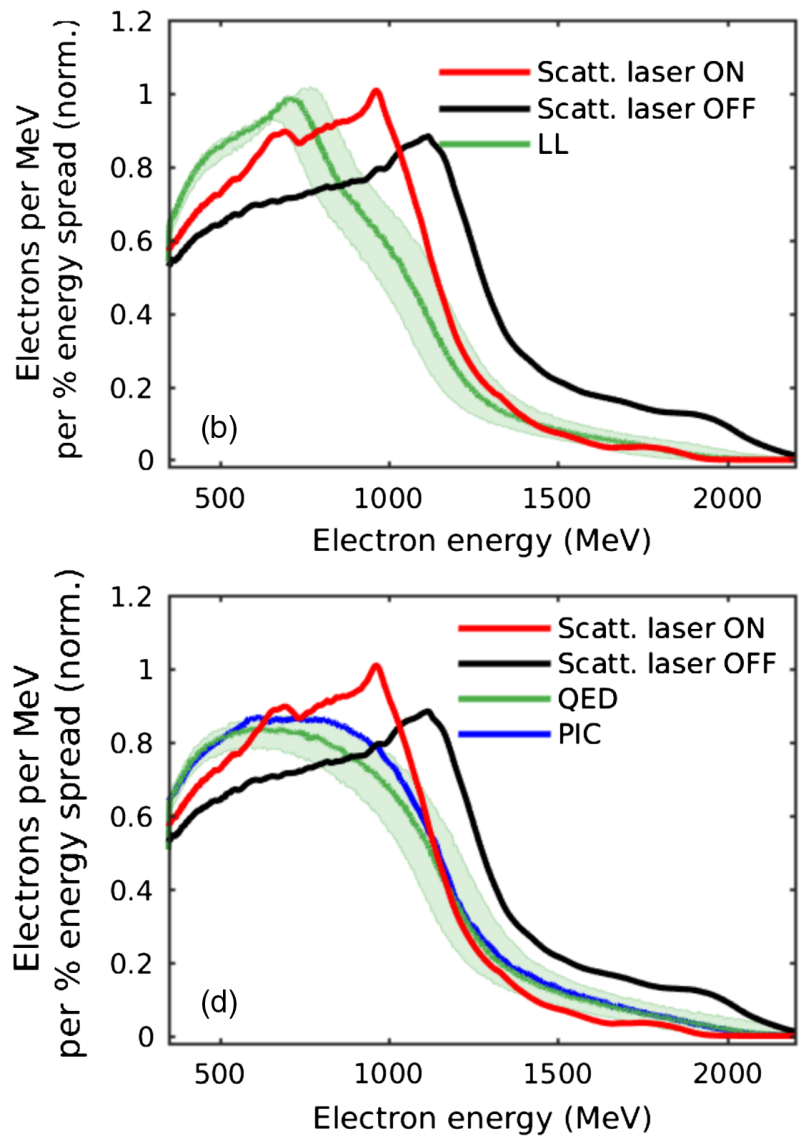

FIG. 4. Comparison of experimental results with theoretical models for the condition of best overlap. The experimentally measured electron spectrum without the scattering laser (black line) and the spectrum of scattered electrons (red line) and (a) the theoretical prediction assuming a model only based on the Lorentz force, (b) the Landau-Lifshitz equation, (c) a semiclassical model of radiation reaction, and (d) the quantum model of radiation reaction in a multiparticle code and in a PIC code (green and blue curves, respectively). In each frame, the uncertainties associated with the theoretical model arise from assuming the experimental uncertainty in the original electron spectrum, as arising from the energy uncertainty of the magnetic spectrometer, and shot-to-shot intensity fluctuations of the scattering laser. Details of the models used are discussed in the text. 
assuming ballistic propagation of the electrons over $1 \mathrm{~cm}$ from a pointlike source. The longitudinal distribution of the electron beam was assumed to be Gaussian with a $12-\mu \mathrm{m}$ FWHM, i.e., 40-fs duration. The transverse laser pulse field profile was instead obtained by fitting the experimental transverse profile [see Fig. 1(b)] with the linear superposition of two Gaussian pulses. Each Gaussian pulse was accurately modeled by including terms up to the fifth order in the diffraction angle. The resulting peak amplitude of the laser field at the focus was $a_{0} \approx 22.5$, with an approximately $2.5-\mu \mathrm{m}$ FWHM of the transverse intensity profile. The laser pulse temporal profile was Gaussian, with a 42-fs duration FMHM of the laser pulse intensity. Since the accelerated electrons lag behind the laser pulse, the head-on collision between the peak of the scattering laser and the peak of the electron beam was set to occur 64 fs after the scattering laser pulse reached the focus. This results in both a reduction of the maximal laser field at the interaction from $a_{0} \approx 22.5$ to $a_{0} \approx 10$ and in an increased diameter (FWHM of the intensity) from 2.5 to about $6.9 \mu \mathrm{m}$ [see Fig. 1(c)].

These simulations were performed assuming different models, associated with different degrees of approximation in modeling RR: a perturbative method [PT, shown in Fig. 4(a)]; the Landau-Lifshitz equation [LL, shown in Fig. 4(b)]; a semiclassical model [SC, shown in Fig. 4(c)]; and a quantum electrodynamic model [QED, shown in Fig. 4(d)]. A discussion of the results predicted by each model is given below.

The PT is routinely used for modeling particle acceleration and transport in synchrotrons [39]. In this case, the electron trajectory in the field is calculated classically using the Lorentz force and the corresponding emitted energy is calculated assuming the relativistic Larmor formula. In this model, the electron energy loss is only accounted for by subtracting the total energy emitted by each electron after the propagation in the field. This model effectively ignores radiation-radiation effects during the propagation of the electron inside the beam. The model significantly fails at reproducing the experimental data for energies approximately below $1.4 \mathrm{GeV}$, as it greatly overestimates the energy loss. This is to be expected, since this model does not account for the continuous energy loss by the electron due to radiation throughout the electron propagation in the laser field and, therefore, predicts a higher emission of radiation.

The predictions of the LL model are shown in Fig. 4(b). It must be noted here that we neglect the term in the equation containing the derivatives of the electromagnetic field [40], since it is negligibly small in our experimental regime and it averages out to zero for a plane-wave pulse [37]. The LL equation is able to reproduce the experimental data more closely, if compared to the PT model, resulting in an overall coefficient of determination $R^{2}=87 \%$. However, this model appears to overestimate the energy loss experienced by the electron beam. Even though the experimental data do not allow us to draw a definite conclusion in this regard, a slight overestimate of the energy loss is to be expected due to the non-negligible value of the quantum parameter $\chi$ in this experiment since, strictly speaking, the LL is valid only under the assumption of $\chi \ll 1$. For non-negligible $\chi$, the LL overestimates the energy loss experienced by the electrons, which results in a spectral peak that is significantly down-shifted if compared with that of the experimental data $(0.78 \pm 0.05 \mathrm{GeV}$ against $0.96 \mathrm{GeV}$ in the experiment). This is because the LL is a purely classical model, with no upper bound in the frequency of the emitted radiation and with continuous emission. In reality, each electron cannot emit a photon with an energy exceeding its kinetic energy, effectively introducing a sharp cutoff in the spectrum of the emitted radiation [10]. This cutoff reduces the total amount of radiation that each electron can emit, thus resulting in a lower energy loss.

This effect of a hard quantum cutoff can be phenomenologically included by multiplying the radiation reaction force in the LL equation by a "weighting" function $g(\chi)=$ $I_{\mathrm{Q}} / I_{C}[41]$, where $I_{\mathrm{Q}}$ is the quantum radiation intensity,

$I_{\mathrm{Q}}=\frac{e^{2} m_{e}^{2}}{3 \sqrt{3} \pi \hbar^{2}} \int_{0}^{\infty} \frac{u\left(4 u^{2}+5 u+4\right)}{(1+u)^{4}} K_{2 / 3}\left(\frac{2 u}{3 \chi}\right) d u$,

and $I_{C}=2 e^{2} m_{e}^{2} \chi^{2} / 3 \hbar^{2}$ is the classical radiation intensity [see Eqs. (4.50) and (4.52) in Ref. [42] ]. In our simulations, the following interpolation formula is employed:

$$
g(\chi) \approx \frac{1}{\left[1+4.8(1+\chi) \ln (1+1.7 \chi)+2.44 \chi^{2}\right]^{2 / 3}},
$$

which approximates the function $g(\chi)$ with accuracy better than $2 \%$ for arbitrary $\chi$ [see Eqs. (4.57) in Ref. [42] ]. With this weighting function, the known classical overestimate of the total emitted energy with respect to the more accurate quantum expression is then avoided. However, in this "semiclassical" model, the emission of radiation is still included as a "classical" continuous process; i.e., the quantum stochastic nature of photon emission is ignored. Moreover, we point out that the used expression of $I_{Q}$ is derived within the so-called local constant cross field approximation, as described in more detail below. A comparison between the predictions of this model and the experimental results is shown in Fig. 4(c). This semiclassical model is able to closely reproduce the experimental data, with an overall coefficient of determination $R^{2}=96 \%$. Indeed, there is agreement for almost all energies, with only a slight deviation around the spectral peak, that is located by the SC model at $0.90 \pm 0.03 \mathrm{GeV}$, and it corresponds to $0.96 \mathrm{GeV}$ in the experiment. However, deviations from the SC model are almost all within $1 \sigma$, and all well within the $2 \sigma$ level. This agreement is significantly better than the one obtained assuming a 
purely classical model based on the LL $\left(R^{2}=87 \%\right)$. This improved agreement of the semiclassical LL model compared to the unmodified LL provides a preliminary indication of the onset of quantum effects under the conditions of the experiment.

Finally, a comparison between the experimentally measured spectrum of the scattered electrons and numerical calculations based on a multiparticle QED code (green curve) is shown in Fig. 4(d). In this model, the stochastic photon emission was calculated for arbitrary electron and photon energies, under the constant cross field approximation. Each electron was propagated according to the Lorentz equation between two consecutive photon emission events [43]. This model is, within the uncertainties of the experiment, able to reproduce the general features of the experimental data. However, there still is a non-negligible mismatch, especially in the shape of the spectral energy density. This mismatch results in a coefficient of determination that is slightly lower $\left(R^{2}=92 \%\right)$ than the semiclassical case.

In order to rule out collective effects in the electron beam as a possible source for this mismatch, three-dimensional PIC simulations using the code EPOCH [44] have also been carried out. For these simulations, the laser and electron bunch simulated were the same as in the multiparticle simulations. The spatial domain extended over $78.7 \mu \mathrm{m}$ in the direction of laser propagation (discretized over 1020 cells) and $40 \mu \mathrm{m}$ in each of the transverse directions (discretized over 920 cells). The collision between the laser pulse and electron bunch occurred $64 \mathrm{fs}$ after the laser pulse reached focus. The electron bunch was represented by $1.5 \times 10^{7}$ macroparticles using third-order particle weighting. The data required to reproduce the PIC simulation results are available in Ref. [45]. Indeed, the PIC and the multiparticle QED model yield very similar results, confirming that collective effects are negligible in our experimental conditions [see Fig. 4(d)].

A possible explanation of this residual mismatch shown by the SC and QED models is a limited validity of the CCFA for our experimental parameters. This approximation is used to calculate the function $g(\chi)$ in the SC model and the probabilities of photon emission in the QED model. The main assumption is that the photon emission is instantaneous or, equivalently, that the formation time of each emitted photon is much smaller than the time where the laser field changes significantly. This allows one to assume a static electromagnetic field during the photon formation process. In order for the CCFA to be valid, we then need the typical temporal variation of the laser field to be much longer than the photon formation time, a reasonable assumption for ultraintense fields (dimensionless laser amplitude $a_{0}$ greatly exceeding 1 ). However, this condition is not necessarily met in our experimental conditions where a peak dimensionless amplitude of $a_{0} \simeq 10$ was reached. The coherence time $\tau_{\mathrm{COH}}$ of the photon in an electric field of magnitude $F_{L}$ can be estimated as [10]

$$
\tau_{\mathrm{COH}} \sim \frac{F_{\mathrm{cr}}}{F_{L}} \frac{\hbar}{m c^{2}}=\frac{1}{a_{0} \omega_{L}}
$$

where $\omega_{L}$ is the laser frequency. On the other hand, the typical temporal variation of the laser electric field is of the order of a quarter of the laser period, i.e., the time it takes the laser electric field to go from zero to its peak value: $\tau_{\text {LASER }} \simeq 0.6$ fs.

Because of the Gaussian temporal profile of the laser intensity, the electron experiences an increasing intensity during its transit through the laser field, resulting in photon formation lengths that are a significant fraction of the typical timescale over which the electric field oscillates. These fractions are of the same order as $1 / a_{0}$, which is not negligible through the laser envelope in our experiment. The CCFA used to obtain radiation reaction in the SC model might then not be strictly valid in our experiment. Indeed, assuming the CCFA for a temporally varying electromagnetic field results in overestimating the energy loss of the electron beam [46], as confirmed by the lower electron energy predicted by the SC when compared with our experimental data. This mismatch is even larger if a QED model based on stochastic photon emission is considered since, in this case, also the photon emission probability relies on the CCFA. In this respect, our experiment suggests that stochasticity effects, which are included in the quantum model but not in the semiclassical model, are less important than effects beyond the CCFA. These preliminary results motivate study of high-field quantum electrodynamics beyond the CCFA, an area of theoretical research that has only recently started to be investigated (see, for instance, Refs. [46,47]).

We have performed a series of simulations, assuming a semiclassical model of RR, in order to check whether a weaker electron energy loss might be attributed to an unaccounted slight transverse misalignment between the electron beam momenta and the direction of propagation of the scattering laser. As an example, a shot with a weaker energy loss [labeled with $c$ in Fig. 2(a)] is well reproduced by the semiclassical calculations if an impact parameter of $5 \mu \mathrm{m}$ is assumed (see Supplemental Material [48]). However, a full parametric study of the transverse misalignment has not been able to compensate for the residual mismatch between theoretical models and experimental data shown in Fig. 4.

As a concluding remark, we must further emphasize that additional potential sources of mismatch might be identified in an incomplete knowledge of the local properties of the laser field, such as its phase content and longitudinal distribution of its intensity. For precise QED testing, these are quantities that must be accurately determined in the focus of a high-intensity laser, which is an extremely challenging task and currently the subject of active research towards the construction of the next generation of ultrahigh-intensity laser facilities. 


\section{CONCLUSIONS AND OUTLOOK}

In conclusion, we report on the experimental detection of strong radiation reaction in an all-optical experiment. The experimental data give clear evidence of significant energy loss $(>30 \%)$ of ultrarelativistic electrons during their interaction with an ultraintense laser field. In their own rest frame, the highest-energy electrons experience an electric field as high as one quarter of the critical field of quantum electrodynamics. The experimental data are best theoretically modeled by taking into account radiation reaction occurring during the propagation of the electrons through the laser field, and best agreement is found for the semiclassical correction of the Landau-Lifshitz equation. The experiment provides a preliminary indication of the limited validity of the constant cross field approximation for our experimental parameters. In order to precisely determine these effects in this class of experiments, several routes can be followed, including fine characterization of the local properties of the laser fields, improved spectral and pointing stability of the electron beam, and narrower energy spectra of the primary electron beam.

The data required to reproduce the PIC simulation results are available in Ref. [45].

\section{ACKNOWLEDGMENTS}

G. Sarri and M. Zepf wish to acknowledge support from the Engineering and Physical Sciences Research Council (EPSRC), UK (Grants No. EP/P010059/1 and No. EP/ N027175/1). C. P. R., J. M. C., and S. P. D. M. acknowledge support from EPSRC (Grants No. EP/M018156/1 and No. EP/M018555/1). K. P., J. M. C., E. G., S.P. D. M., and Z. N. acknowledge funding from STFC (ST/J002062/ 1 and ST/P002021/1). A. T. and K. B. acknowledge support from the U.S. NSF CAREER Grant No. 1054164 and A. T., K. B., and K.K. from the U.S. DOD Grant No. W911NF1610044 and U.S. DOE Grant No. DENA0002372. All the authors acknowledge the technical support from the Central Laser Facility.

[1] L. D. Landau and E. M. Lifshitz, The Classical Theory of Fields (Butterworth-Heinemann, Amsterdam, 2000), paragraph 76.

[2] V.S. Krivitskii and V. N. Tsytovich, Average RadiationReaction Force in Quantum Electrodynamics, Sov. Phys. Usp. 34, 250 (1991).

[3] A. Di Piazza, C. Müller, K. Z. Hatsagortsyan, and C. H. Keitel, Extremely High-Intensity Laser Interactions with Fundamental Quantum Systems, Rev. Mod. Phys. 84, 1177 (2012) and references therein.

[4] M. Chen, E. Esarey, C. G. R. Geddes, C. B. Schroeder, G. R. Plateau, S. S. Bulanov, S. Rykovanov, and W. P. Leemans, Modeling Classical and Quantum Radiation from LaserPlasma Accelerators, Phys. Rev. ST Accel. Beams 16, 030701 (2013).
[5] N. Neitz and A. Di Piazza, Stochasticity Effects in Quantum Radiation Reaction, Phys. Rev. Lett. 111, 054802 (2013).

[6] T. G. Blackburn, C. P. Ridgers, J. G. Kirk, and A. R. Bell, Quantum Radiation Reaction in Laser-Electron Beam Collisions, Phys. Rev. Lett. 112, 015001 (2014).

[7] M. Vranic, T. Grismayer, R. A. Fonseca, and L. O. Silva, Quantum Radiation Reaction in Head-On Laser-Electron Beam Interaction, New J. Phys. 18, 073035 (2016).

[8] V. Dinu, C. Harvey, A. Ilderton, M. Marklund, and G. Torgrimsson, Quantum Radiation Reaction: From Interference to Incoherence, Phys. Rev. Lett. 116, 044801 (2016).

[9] V. B. Berestetskii, E. M. Lifshitz, and L. P. Pitaevskii, Quantum Electrodynamics (Elsevier Butterworth-Heinemann, Oxford, 1982).

[10] V. I. Ritus, Quantum Effects of the Interaction of Elementary Particles with an Intense Electromagnetic Field, J. Russ. Laser Res. 6, 497 (1985).

[11] R. Ruffini, G. Vereshchagin, and S. Xue, Electron-Positron Pairs in Physics and Astrophysics: From Heavy Nuclei to Black Holes, Phys. Rep. 487, 1 (2010).

[12] J. Sultana, D. Kazanas, and A. Mastichiadis, The Supercritical Pile Gamma-Ray Burst Model: The GRB Afterglow Steep-Decline-and-Plateau Phase, Astrophys. J. 779, 16 (2013).

[13] G. A. Mourou, G. Korn, W. Sandner, and J. L. Collier, Extreme Light Infrastructure: Science and Technology with Ultra-Intense Lasers WHITEBOOK (THOSS Media, $\mathrm{GmbH}$, Berlin, 2011).

[14] I. C. E. Turcu et al., High Field Physics and QED Experiments at ELI-NP, Romanian reports in Physics 68, S145 (2016).

[15] J. P. Zou et al., High Power Laser Science and Engineering Design and Current Progress of the Apollon 10 PW Project, High Power Laser Sci. Eng. 3, e2 (2015).

[16] http://spie.org/x103688.xml.

[17] http://www.xcels.iapras.ru/2013_news.html.

[18] E. Esarey, C. Schroeder, and W. Leemans, Physics of LaserDriven Plasma-Based Electron Accelerators, Rev. Mod. Phys. 81, 1229 (2009) and references therein.

[19] W.P. Leemans et al., Multi-GeV Electron Beams from Capillary-Discharge-Guided Subpetawatt Laser Pulses in the Self-Trapping Regime, Phys. Rev. Lett. 113, 245002 (2014).

[20] H. T. Kim, K. H. Pae, H. J. Cha, I. J. Kim, T. J. Yu, J. H. Sung, S. K. Lee, T. M. Jeong, and J. Lee, Enhancement of Electron Energy to the Multi-GeV Regime by a Dual-Stage Laser-Wakefield Accelerator Pumped by Petawatt Laser Pulses, Phys. Rev. Lett. 111, 165002 (2013).

[21] X. Wang et al., Quasi-monoenergetic Laser-Plasma Acceleration of Electrons to $2 \mathrm{GeV}$, Nat. Commun. 4, 1988 (2013).

[22] G. Sarri et al., Ultrahigh Brilliance Multi-MeV Gamma-Ray Beams from Nonlinear Relativistic Thomson Scattering, Phys. Rev. Lett. 113, 224801 (2014).

[23] K. Khrennikov, J. Wenz, A. Buck, J. Xu, M. Heigoldt, L. Veisz, and S. Karsch, Tunable All-Optical Quasimonochromatic Thomson X-Ray Source in the Nonlinear Regime, Phys. Rev. Lett. 114, 195003 (2015).

[24] W. Yan et al., High-Order Multiphoton Thomson Scattering, Nat. Phot. 11, 514 (2017). 
[25] C. Bula et al., Observation of Nonlinear Effects in Compton Scattering, Phys. Rev. Lett. 76, 3116 (1996).

[26] D. L. Burke et al., Positron Production in Multiphoton Light-by-Light Scattering, Phys. Rev. Lett. 79, 1626 (1997).

[27] K. Poder et al., Multi-GeV Electron Acceleration in Wakefields Strongly Driven by Oversized Laser Spots (to be published).

[28] J. M. Cole et al. Experimental Evidence of Radiation Reaction in the Collision of a High-Intensity Laser Pulse with a Laser-Wakefield Accelerated Electron Beam, Phys. Rev. X 8, 011020 (2018).

[29] S. Kneip et al., Bright Spatially Coherent Synchrotron X-rays from a Table-Top Source, Nat. Phys. 6, 980 (2010).

[30] G. M. Samarin, M. Zepf, and G. Sarri, Radiation Reaction Studies in an All-Optical Set-up: Experimental Limitations, J. Mod. Opt. 65, 1362 (2017).

[31] J. Osterhoff et al., Generation of Stable, Low-Divergence Electron Beams by Laser-Wakefield Acceleration in a Steady-State-Flow Gas Cell, Phys. Rev. Lett. 101, 085002 (2008).

[32] M. Vargas, W. Schumaker, Z.-H. He, Z. Zhao, K. Behm, V. Chvykov, B. Hou, K. Krushelnick, A. Maksimchuk, V. Yanovsky, and A. G. R. Thomas, Improvements to Laser Wakefield Accelerated Electron Beam Stability, Divergence, and Energy Spread Using Three-Dimensional Printed Two-Stage Gas Cell Targets, Appl. Phys. Lett. 104, 174103 (2014).

[33] C. Gregory (private communication).

[34] D. J. Corvan, T. Dzelzainis, C. Hyland, G. Nersisyan, M. Yeung, M. Zepf, and G. Sarri, Optical Measurement of the Temporal Delay Between Two Ultra-short and Focussed Laser Pluses, Opt. Express 24, 3127 (2016).

[35] G. Battistoni, F. Cerutti, A. Fassò, A. Ferrari, S. Muraro, J. Ranft, S. Roesler, and P.R. Sala, The FLUKA Code: Description and Benchmarking, AIP Conf. Proc. 896, 31 (2007).

[36] S. Corde, K. Ta Phuoc, G. Lambert, R. Fitour, V. Malka, A. Rousse, A. Beck, and E. Lefebvre, Femtosecond $X$ rays from Laser-Plasma Accelerators, Rev. Mod. Phys. 85, 1 (2013).

[37] A. Di Piazza, Exact Solution of the Landau-Lifshitz Equation in a Plane Wave, Lett. Math. Phys. 83, 305 (2008).

[38] A. G. R. Thomas, C. P. Ridgers, S. S. Bulanov, B. J. Griffin, and S. P. D. Mangles, Strong Radiation-Damping Effects in a Gamma-Ray Source Generated by the Interaction of a High-Intensity Laser with a Wakefield-Accelerated Electron Beam, Phys. Rev. X 2, 041004 (2012).

[39] G. Stupakov and S. Heifets, Beam Instability and Microbunching Due to Coherent Synchrotron Radiation, Phys. Rev. ST Accel. Beams 5, 054402 (2002).

[40] M. Tamburini, F. Pegoraro, A. Di Piazza, C. H. Keitel, and A. Macchi, Radiation Reaction Effects on Radiation Pressure Acceleration, New J. Phys. 12, 123005 (2010).

[41] J. G. Kirk, A. R. Bell, and I. Arka, Pair Production in Counter-propagating Laser Beams, Plasma Phys. Controlled Fusion 51, 085008 (2009).

[42] V. N. Baier, V. M. Katkov, and V. M. Strakhovenko, Electromagnetic Processes at High Energies in Oriented Single Crystals (World Scientific, Singapore, 1998).

[43] M. Tamburini, A. Di Piazza, and C. H. Keitel, Laser-PulseShape Control of Seeded QED Cascades, Sci. Rep. 7, 5694 (2017). See also arXiv:1511.03987.

[44] T. D. Arber, K. Bennett, C. S. Brady, A. Lawrence-Douglas, M. G. Ramsay, N. J. Sircombe, P. Gillies, R. .G Evans, H. Schmitz, A. R. Bell, and C.P. Ridgers, Contemporary Particle-in-Cell Approach to Laser-Plasma Modelling, Plasma Phys. Controlled Fusion 57, 113001 (2015).

[45] K. Poder et al., Data for "Experimental Signatures of the Quantum Nature of Radiation Reaction in the Field of an Ultraintense Laser," DOI: 10.15124/bd4a4a0b-78e3-405c9318-1757b0209c54 (2018).

[46] A. Di Piazza, M. Tamburini, S. Meuren, and C. H. Keitel, Implementing Nonlinear Compton Scattering beyond the Local Constant Field Approximation, arXiv:1708.08276.

[47] C. N. Harvey, A. Ilderton, and B. King, Testing Numerical Implementations of Strong-Field Electrodynamics, Phys. Rev. A 91, 013822 (2015).

[48] See Supplemental Material at http://link.aps.org/ supplemental/10.1103/PhysRevX.8.031004 for a comparison between the experimental spectra and the ones simulated using the semi-classical model. A transverse misalignment between the laser and the electron beam main axis of 5 microns is assumed.

Correction: The data availability statement has now been relocated and anchored with complete source information. 